\title{
PARATHYROID ADENOMA PRESENTING AS PRIMARY HYPERPARATHYROIDISM
}

\author{
Amit K. Chopraㅁ, Vijinder Arora ${ }^{2}$, Inderbir S. Nijjar³, Rajiv Sharma ${ }^{4}$
}

\section{HOW TO CITE THIS ARTICLE:}

Amit K. Chopra, Vijinder Arora, Inderbir S. Nijjar, Rajiv Sharma. "Parathyroid Adenoma presenting as Primary Hyperparathyroidism". Journal of Evolution of Medical and Dental Sciences 2014; Vol. 3, Issue 08, February 24; Page: 1920-1923, DOI: $10.14260 /$ jemds/2014/2080

INTRODUCTION: Excessive production of PTH, termed hyperparathyroidism, is classified as primary, secondary, or tertiary in form. Primary hyperparathyroidism usually occurs in the setting of a parathyroid adenoma (80\%) but can also be seen with parathyroid gland hyperplasia (15\%-20\%) or carcinoma $(<0.5 \%){ }^{1}$ Although most patients with primary hyperparathyroidism today are asymptomatic due to the widespread availability of laboratory screening for hypercalcemia and earlier detection, the clinical presentation is variable ${ }^{2}$.

We discuss a case of 42 years old female, who was diagnosed to be a case of primary hyperparathyroidism due to a parathyroid adenoma in neck.

CASE REPORT: A 42 years old female presented to us with complaints of constipation, bone pains and nausea for the last few months. There was no history of any renal stones, polyuria or any loss of consciousness in the past. On examination, she was $158 \mathrm{~cm}$ in height and $68 \mathrm{~kg}$ in weight. Her BP was $124 / 78 \mathrm{~mm} \mathrm{Hg}$ and her pulse was 78/min. There was no anemia, jaundice, clubbing or any neck swelling on palpation. No other abnormal finding was observed in the chest or abdomen on systemic examination on routine laboratory investigations, her serum calcium was raised at $12.5 \mathrm{mg} / \mathrm{dl}$ in addition to her serum parathyroid levels being raised upto $324.30 \mathrm{pg} / \mathrm{ml}$ (normal 15-68 pg/ml).

Her serum alkaline phosphatase levels were also found be raised. Vitamin D levels were normal. There was no abnormality in her thyroid hormone levels.

Her ECG showed normal sinus rhythm and her chest x-ray was unremarkable. Her ultrasound neck was got done which revealed a mass near upper pole of thyroid gland. Patient was then taken up for MRI neck which revealed a well-defined, oval shaped mass posterior to the left lobe of thyroid near its upper pole (Fig. 1). It was isointense on T1 W images and hypointense on T2W images. It measured $1.8 \times 1.5 \times 1.1 \mathrm{~cm}$ in size. It was indenting the posterior margin of thyroid and was abutting the esophagus medially and internal carotid laterally (Fig. 2). Her sestamibi scan showed presence of parathyroid adenoma (Fig. 4).

The patient is on conservative treatment right now including fluid resuscitation, diuretics and bisphosphonate therapy and is awaiting surgery.

DISCUSSION: Primary hyperparathyroidism, in approximately $80 \%$ of cases, is caused by a single parathyroid adenoma, and rarely by multiple adenoma or hyperplasia, in 1-2\% of cases the cause could be carcinoma. ${ }^{3}$ The incidence of primary hyperparathyroidism is approximately 1 per 1,000 people $(0.1 \%){ }^{4}$

In minority of cases, this occurs as a part of multiple endocrine neoplasia (MEN) syndrome either type 1 or type $2 \mathrm{a}$. 
Secondary hyperparathyroidism is due to a physiological secretion of parathyroid hormone (PTH) by the parathyroid glands in response to hypocalcemia. The most common causes are vitamin D deficiency and chronic renal failure.

Tertiary hyperparathyroidism is seen in patients with long-term secondary hyperparathyroidism which eventually leads to hyperplasia of parathyroid glands and a loss of response to serum calcium levels. This disorder is commonly seen in chronic renal failure patients and is an autonomous activity.

Based on the pathophysiology of primary hyperparathyroidism, increased secretion of PTH promotes a release of calcium by inhibition of osteoblasts and stimulating osteoclasts. In the kidneys, PTH causes a decrease in calcium clearance, and increases renal excretion of phosphate by inhibiting its reabsorption in the tubules. PTH stimulates hydroxylation of 25-hydroxy vitamin D to 1, 25dihydroxy vitamin D in the kidneys; it is the latter which causes enhanced absorption of calcium in the intestine.

Some common symptoms and their prevalence in these patients are as follows: Nephrolithiasis (30\%), bone disease (2\%), peptic ulcer disease (12\%), psychiatric disorders (15\%), muscle weakness (70\%), constipation (32\%), polyuria (28\%), pancreatitis (1\%), myalgia (54\%) and arthralgia (54\%); 2 the clue to the diagnosis of primary hyperparathyroidism is usually the laboratory finding of hypercalcemia.

Previously encountered overt symptoms are no longer common. Patients now often complain of mild subjective symptoms such as weakness and easy fatigability. As mentioned in our case, patient was not having very specific clinical findings except of bone pains, which are quite common in hyperparathyroidism.

Generalized osteopenia is the most common imaging finding in primary hyperparathyroidism. Subperiosteal bone resorption is a common finding of advanced hyperparathyroidism, most often seen involving the hands and the feet. Brown tumors, also known as osteoclastomas, are eccentrically located and often expansile lesions resulting from amassing osteoclasts and fibrous tissue. Although also having a higher prevalence in primary hyperparathyroidism, brown tumors are more frequently encountered in the setting of secondary hyperparathyroidism due to the greater prevalence of this condition Soft-tissue calcification and periostitis are also commonly seen in secondary hyperparathyroidism.

The gold standard of diagnosis is the Parathyroid immunoassay. Once an elevated Parathyroid hormone has been confirmed, goal of diagnosis is to determine whether the hyperparathyroidism is primary or secondary in origin by obtaining a serum calcium level. Although directed US examination, CT of the neck, and magnetic resonance imaging are useful in the complete evaluation of primary hyperparathyroidism, ${ }^{99 \mathrm{~m} T c-s e s t a m i b i}$ parathyroid scintigraphy is now considered the best preoperative localizing modality for the detection of parathyroid adenomas.

As most patients with primary hyperparathyroidism are asymptomatic, treatment is controversial. The National Institutes of Health (NIH) consensus statement in 1990 concluded that while surgical intervention is the acceptable treatment of primary hyperparathyroidism, "conscientious surveillance may be justified in patients whose calcium levels are only mildly elevated and whose renal and bone status are close to normal." NIH criteria identified for selecting patients for parathyroidectomy include the following: (a) typical parathyroid-related symptoms involving the skeletal, renal, or gastrointestinal systems; (b) a sustained elevation of serum calcium level more 
than 1-1.6 mg/dL (0.25-0.40 mmol/L) above the upper limits of normal; (c) a substantial decline in bone mass; (d) a decline in renal function by 30\% or more; (e) nephrolithiasis or worsening of calciuria; ( $f$ ) severe neuromuscular or psychological problems; or ( $g$ ) unwillingness of the patient to continue under medical supervision. ${ }^{5}$

Patients who on routine testing are found to have a mildly elevated serum calcium concentration (2.65-2.75 mmol/L) may be treated conservatively. If surgery is not to be performed, these patients should be monitored regularly with serum calcium every 6 months and 24 hour urinary calcium excretion and bone density every 12 months. Most patients do not get worse over years of follow-up care. Estrogen and bisphosphonate therapies may reduce some of the PTH effects of the disease, but will not directly control glandular overactivity. Estrogen and alendronate (fosamax) are important therapies for women with osteoporosis and primary hyperparathyroidism. These agents may increase bone densities by $4-6 \%$ over 2 years.

A calcimimetic (cinacalcet) is a new type of drug to be considered as a potential therapy for some people with severe hypercalcemia and primary hyperparathyroidism who are unable to undergo parathyroidectomy and for secondary hyperparathyroidism on dialysis.

\section{REFERENCES:}

1. Lenchik L, Mitchell K. Hyperparathyroidism and renal osteodystrophy. In: El-Khoury G, ed. Essentials of musculoskeletal imaging. Philadelphia, Pa: Churchill Livingstone, 2003; 295-301.

2. Genant HK, Hick LL, Lanzl LH, Rossmann K, Horst JV, Paloyan E. Primary hyperparathyroidism: a comprehensive study of clinical, biochemical, and radiographic manifestations. Radiology 1973;109:513-24.

3. John T, Potts Jr. Disease of the parathyroid gland and other hyper and hypocalcemic disorders. In: Fauci AS, Braunwald E, Isselbacher KJ,Wilson JD, Martin JB, Kasper DL, editors. Harrison's principles of internal medicine. 16th ed. New York: Mc Graw -Hill Co; 2005. p. 2249-67.

4. Deshmukh, R. G.; Alsagoff, S. A. L.; Krishnan, S.; Dhillon, K. S.; Khir, A.S. M. (1998). "Primary hyperparathyroidism presenting with pathological fracture". Journal of the Royal College of Surgeons of Edinburgh 43 (6):424-27.

5. NIH conference. Diagnosis and management of asymptomatic primary hyperparathyroidism: consensus development conference statement. Ann Intern Med 1991; 114:593-97.

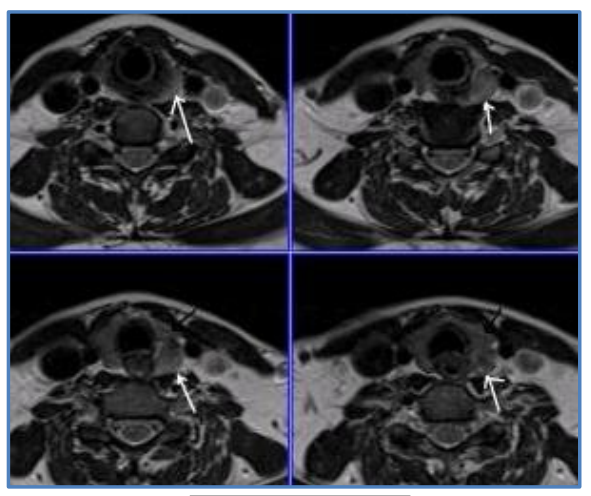

Figure 1

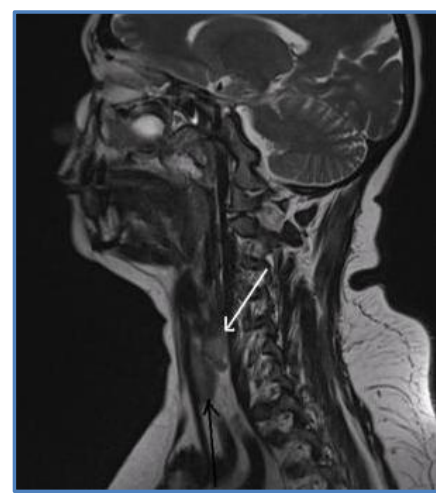

Figure 2 


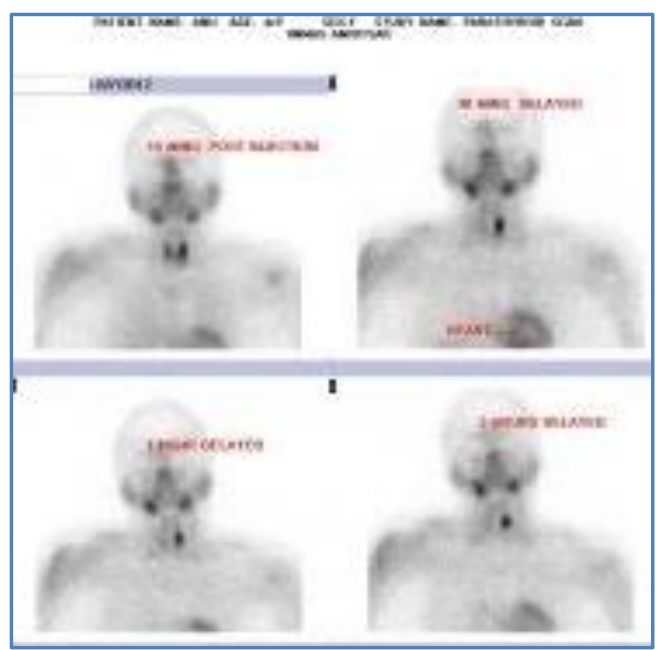

Figure 4

\section{AUTHORS:}

1. Amit K. Chopra

2. Vijinder Arora

3. Inderbir S. Nijjar

4. Rajiv Sharma

\section{PARTICULARS OF CONTRIBUTORS:}

1. Assistant Professor, Department of Community Medicine, SGRDIMSR, Amritsar.

2. Consultant Radiologist, Department of Radiology, Nijjar Scan Centre, Amritsar.

3. Consultant Radiologist, Department of Radiology, Nijjar Scan Centre, Amritsar.

4. Assistant Professor, Department of Medicine, Guru Nanak Dev Hospital, Amritsar.
NAME ADDRESS EMAIL ID OF THE CORRESPONDING AUTHOR:

Dr. Amit K. Chopra,

House No. 11, Lane 2, Gopal Nagar, Majitha Road, Opposite Samosa Halwai, Amritsar.

E-mail: anginaplus@yahoo.com

Date of Submission: 28/01/2014.

Date of Peer Review: 29/01/2014.

Date of Acceptance: 06/02/2014.

Date of Publishing: 19/02/2014. 